\title{
Platelet-induced Neurogenic Coronary Contractions Due to Accumulation of the False Neurotransmitter, 5-Hydroxytryptamine
}

Richard A. Cohen

With the technical assistance of Kevin Zitnay and Robert Weisbrod

Robert Dawson Evans Memorial Department of Clinical Research, Peripheral Vascular Section, University Hospital E411,

Boston University Medical Center, Boston, Massachusetts 02118

\section{Abstract}

The purpose of this study was to determine if 5-hydroxytryptamine released from aggregating platelets could be accumulated and released by canine coronary adrenergic nerves, and if the false neurotransmitter resulted in an abnormal response of the smooth muscle to nerve stimulation. Isometric tension was measured in rings of epicardial coronary arteries suspended in organ chambers filled with physiological salt solution. The response to electrical stimulation or exogenously added norepinephrine was elicited after contraction with prostaglandin $F_{2 \alpha}$. Electrical stimulation and exogenous norepinephrine caused beta-adrenergic relaxation of control rings. However, after rings were exposed for $2 \mathrm{~h}$ to aggregating platelets or 5-hydroxytryptamine, electrical stimulation caused frequency-dependent contractions. These contractions were prevented by the serotonergic antagonists, cyproheptadine or ketanserin, or by the neuronal uptake inhibitor, cocaine. The relaxation caused by exogenously added norepinephrine was unchanged after exposure to platelets or 5-hydroxytryptamine, indicating that smooth muscle alpha- and beta-adrenergic responsiveness was unchanged. The electrically stimulated overflow of radiolabeled norepinephrine from superfused strips of coronary artery was not altered by prior exposure to 5-hydroxytryptamine, indicating that the effect of exposure on the response to electrical stimulation is primarily at smooth muscle serotonergic receptors.

Canine coronary arteries accumulated and metabolized radiolabeled 5-hydroxytryptamine in vitro. The accumulation of 5-hydroxytryptamine was inhibited by cocaine or by adrenergic denervation with 6-hydroxydopamine but unaffected by removal of endothelium, indicating that the adrenergic nerves were the primary site of accumulation. Electrical stimulation of superfused strips of coronary artery preincubated with radiolabeled 5-hydroxytryptamine caused the release of the intact indoleamine; this was blocked by the neurotoxin, tetrodotoxin. These studies suggest that 5-hydroxytryptamine liberated from aggregating platelets may be accumulated by coronary adrenergic nerve endings. Upon its release from the nerves as a false transmitter, the amine can activate serotonergic receptors on the smooth muscle and reverse the action of the adrenergic nerves from dilator to constrictor.

Received for publication 18 June 1984 and in revised form 29 August 1984.

J. Clin. Invest.

(c) The American Society for Clinical Investigation, Inc.

0021-9738/85/01/0286/07 \$1.00

Volume 75, January 1985, 286-292

\section{Introduction}

Aggregating platelets contract isolated epicardial canine coronary arteries (1). Since these contractions are due largely to 5-hydroxytryptamine $(5-\mathrm{HT})^{1}$ released by the platelets, the amine can be implicated in spasm of coronary arteries occurring at sites where platelets aggregate (1-3). It is possible that 5-HT liberated by platelets can also influence the function of coronary adrenergic nerves. Since norepinephrine released by adrenergic nerves causes beta-adrenergic relaxation of coronary smooth muscle (4), interference with neural function might promote coronary spasm. It is known that $5-\mathrm{HT}$ may be accumulated by adrenergic nerve terminals in nonvascular $(5,6)$ and vascular smooth muscle $(7,8)$, and that after its accumulation the amine may be released upon stimulation of the nerves. It is not known if neurally released 5-HT alters the function of adrenergic nerves. The purpose of this study was to determine if 5-HT released by aggregating platelets could be accumulated by canine coronary adrenergic nerves and if the amine could act as a false neurotransmitter to modify the response of coronary smooth muscle to subsequent nerve stimulation.

\section{Methods}

The heart was removed from mongrel dogs after anesthesia with sodium pentobarbital $(30 \mathrm{mg} / \mathrm{kg}$, i.v.) and exsanguination; the epicardial coronary arteries were immediately dissected free.

Organ bath experiments. $6 \mathrm{~mm}$ long rings of left circumflex artery and its first major epicardial ventricular branch were placed in organ chambers $(25 \mathrm{ml})$ filled with physiological salt solution of the following millimolar composition: $\mathrm{NaCl}, 118.3 ; \mathrm{KCl}, 4.7 ; \mathrm{MgSO}_{4}, 1.2 ; \mathrm{KH}_{2} \mathrm{PO}_{4}$, 1.2; $\mathrm{CaCl}_{2}, 2.5 ; \mathrm{NaHCO}_{3}, 25.0$; calcium EDTA, 0.026; and glucose, 11.1. The solution was maintained at $37^{\circ} \mathrm{C}$ and gassed with $95 \% \mathrm{O}_{2}-$ $5 \% \mathrm{CO}_{2}$. The rings were connected to a strain gauge for measurement of isometric circumferential force. Over $90 \mathrm{~min}$, length of the smooth muscle was gradually increased to adjust basal tension to that previously found to be optimal for contraction (4); thereafter, length was not altered. In some rings the endothelium was mechanically denuded; its absence was demonstrated by failure of the ring contracted in the presence of prostaglandin $F_{2 \alpha}$ to relax in response to acetylcholine $\left(10^{-6} \mathrm{M} ;[1,9]\right)$. Transmural electrical field stimulation of coronary artery rings was accomplished with parallel platinum electrodes, a stimulator, and a d.c. amplifier. Optimal neurogenic stimulation parameters were determined in each experiment as previously described, and all experiments were performed in the presence of indomethacin $\left(3 \times 10^{-5} \mathrm{M} ;[4]\right)$. To study relaxation in response to electrical stimulation, rings were contracted with prostaglandin $\mathrm{F}_{2 \alpha}\left(2 \times 10^{-6} \mathrm{M}\right)$. Responses to each frequency of stimulation $(0.5-16 \mathrm{~Hz})$ were allowed to reach maximum before the frequency was increased. Unless otherwise

1. Abbreviations used in this paper: 5-HT, 5-hydroxytryptamine; 5-HIAA, 5-hydroxyindole acetic acid; IC $_{50}$, concentration causing $50 \%$ relaxation. 
noted, the change in tension caused by stimulation was expressed as a percentage of the contraction induced by prostaglandin $F_{2 \alpha}(4)$.

The prostaglandin $F_{2 \alpha}$-induced tension was allowed to stabilize for $20 \mathrm{~min}$ after each response to electrical stimulation. The response of the rings to cumulative half $\log$ unit increments in bath concentration of norepinephrine $\left(10^{-9}\right.$ to $\left.10^{-6} \mathrm{M}\right)$ was then determined as described (4). Relaxations in response to norepinephrine were expressed as a percentage of the maximal contraction of the ring to prostaglandin $F_{2 \alpha}$. The concentration that caused $50 \%$ relaxation was estimated by graphical interpolation and is expressed as the logarithm $\left(\log \mathrm{IC}_{50}\right)$.

Some vessels were stored in physiological salt solution at $4^{\circ} \mathrm{C}$ for up to $24 \mathrm{~h}$ after their dissection from the heart. As compared with fresh vessels, stored vessels contracted significantly less in response to prostaglandin $F_{2 \alpha}(4.1 \pm 0.9 \mathrm{~g}[n=6]$ vs. $7.8 \pm 0.9 \mathrm{~g}[n=6], P<0.05)$. However, the responses to electrical stimulation, and that to norepinephrine, expressed as a percentage of prostaglandin $F_{2 \alpha}$-induced tension were not significantly different, and the $\log \mathrm{IC}_{50}$ of exogenously added norepinephrine was unchanged (stored, $-7.1 \pm 0.2$; fresh, -7.2 +0.1 ). The data from fresh and stored vessels were therefore combined.

Platelets and 5-HT. Autologous blood was collected in modified acid/citrate/dextrose anticoagulant $(1,2)$. Platelet-rich plasma was obtained by centrifugation $(180 \mathrm{~g}, 20 \mathrm{~min})$. A platelet pellet was obtained $(1,600 \mathrm{~g}, 10 \mathrm{~min})$ and resuspended in calcium-free saline containing $0.4 \%$ citrate at $\mathrm{pH}$ 6.5. The number of platelets in these suspensions was counted with a Coulter counter (Coulter Instruments, Inc., Hialeah, FL) and suspensions were added to the physiological salt solution in the organ chamber, resulting in a final concentration of $7.0 \pm 1.2 \times 10^{4}$ platelets $/ \mu \mathrm{l}(n=12)$. After control responses to electrical stimulation and exogenous norepinephrine were obtained, coronary rings were exposed to aggregating platelets or 5- $\mathrm{HT}\left(10^{-6} \mathrm{M}\right)$ added to the organ chamber for $2 \mathrm{~h}$; the platelets or 5-HT solution was replaced once after the first hour. The effect of platelets was compared with that of a supernatant obtained by centrifugation $(1,600$ $g, 10 \mathrm{~min}$ ) of the concentrated platelet suspension. Indomethacin was omitted from the physiological salt solution during the addition of platelets; spontaneous aggregation occurred as evidenced by clearing of the initially turbid solution and absence of a pellet upon centrifugation of the bath contents $(1,2)$. As previously reported, addition of aggregating platelets and 5-HT, but not of the platelet supernatant, caused contractions of the coronary rings $(1,2)$. After this exposure the rings were repeatedly washed for $1 \mathrm{~h}$ during which tension returned to base line; responses to electrical stimulation and exogenous norepinephrine were then repeated after contraction by prostaglandin $F_{2 \alpha}(2$ $\times 10^{-6} \mathrm{M}$ ). Neither the exposure to platelets nor that to 5-HT significantly changed the contractions caused by prostaglandin $F_{2 a}$ as compared with those observed before exposure. (Platelets: before, $8.5 \pm 0.9 \mathrm{~g}$; after, $8.8 \pm 1.4 \mathrm{~g}$; 5-HT: before, $6.9 \pm 1.0 \mathrm{~g}$; after, $7.2 \pm 1.3 \mathrm{~g}$.) The effects of antagonists on responses of the rings after exposure to platelets or 5HT were determined after incubation with the antagonists for $30 \mathrm{~min}$. The effect of neuronal uptake blockade by cocaine $\left(3 \times 10^{-5} \mathrm{M}\right)$ on the effects of exposure to $5-\mathrm{HT}\left(10^{-6} \mathrm{M}\right)$ was determined in pairs of rings. One ring was treated with cocaine for $15 \mathrm{~min}$ before the 2-h exposure to 5-HT; the other was treated with cocaine after the exposure to 5-HT. Since cocaine itself increased the relaxation in response to electrical stimulation, the responses of these two rings were further compared with those of rings exposed to control solution or cocaine alone but stimulated simultaneously with those exposed to 5-HT. Neither the antagonists nor cocaine significantly affected the contractions caused by prostaglandin $F_{2 \alpha}$.

5-HT exposure and stimulated overflow of radiolabeled norepinephrine. To determine the effect of 5-HT exposure on the neuronal release of norepinephrine, longitudinal strips of coronary arteries $(\sim 2 \mathrm{~cm}$ long, 26-105 mg) were incubated for $60 \mathrm{~min}$ in $\left[{ }^{3} \mathrm{H}\right]$ norepinephrine $\left(10^{-7} \mathrm{M}\right)$ and superfused as previously described $(4,10)$. In brief, the strips were rinsed in fresh physiological salt solution and suspended under $3.5 \mathrm{~g}$ tension between two platinum wires for electrical stimulation. The strips were superfused at $3 \mathrm{ml} / \mathrm{min}$ with oxygenated physiological salt solution at $37^{\circ} \mathrm{C}$. After an initial washout period of $120 \mathrm{~min}$, the superfusate was collected for 2-min intervals for determination of the overflow from the tissue of radioactive compounds. The strips were electrically stimulated for $6 \mathrm{~min}$ at $2 \mathrm{~Hz}(10 \mathrm{~V}, 0.2$ $\mathrm{ms})$, and thereafter superfused with control solution for $3 \mathrm{~h}$ or with 5-HT $\left(10^{-6} \mathrm{M}\right)$ for $2 \mathrm{~h}$ followed by a 1-h washout before a second 6-min stimulation. At the conclusion of the experiments, the strips were blotted dry, weighed, and the tritiated compounds were then extracted with $1 \mathrm{~N}$ acetic acid containing $0.03 \mathrm{mM}$ disodium EDTA and $5 \mathrm{mM}$ ascorbic acid $(11,12)$. The tritiated compounds overflowing the tissue were quantitated as fraction released, calculated as the ratio of disintegrations per minute released per unit time to the total disintegrations per minute extracted plus disintegrations per minute released. In portions of the samples collected before and during each stimulation, tritiated norepinephrine was separated from its major metabolites as described previously $(4,10-12)$. Evoked overflow of $\left[{ }^{3} \mathrm{H}\right]$ norepinephrine was calculated as the overflow per milligram tissue during the 6-min stimulation minus that during the preceding $6 \mathrm{~min}$.

Accumulation of radiolabeled 5-HT. Segments of epicardial coronary arteries ( $2 \mathrm{~cm}$ long, 22-72 $\mathrm{mg})$ were incubated for $2 \mathrm{~h}$ in $\left[{ }^{14} \mathrm{C}\right]-5$ HT $\left(10^{-6} \mathrm{M}\right)$. Some segments were previously denuded of endothelium (3). Other segments were denervated by twice exposing them to 6-hydroxydopamine $\left(0.3 \mathrm{mg} / \mathrm{ml}, 10\right.$ minutes, at $\left.\mathrm{pH} 4.9,37^{\circ} \mathrm{C} ; 13\right)$. In additional preparations, neuronal uptake was blocked by a 15 -min pretreatment with cocaine $\left(3 \times 10^{-5} \mathrm{M}\right)$. At the end of the incubation with $\left[{ }^{14} \mathrm{C}\right] 5-\mathrm{HT}$, the radiolabeled compounds were extracted at $4^{\circ} \mathrm{C}$ for $1 \mathrm{~h}$ in two $2.5-\mathrm{ml}$ aliquots of $0.1 \mathrm{~N} \mathrm{HCl}$ containing ascorbic acid $(0.1 \mathrm{mg} / \mathrm{ml})(7)$. The pooled extraction media contained $95 \pm 1.0 \%(n$ $=8$ ) of tritiated compounds as determined after tissue solubilization in Solulyte (J. T. Baker Chemical Co., Phillipsburg, NJ). The aliquots were pooled and frozen until intact radiolabeled 5-HT was determined chromatographically.

Overflow of radiolabeled 5-HT. Longitudinal strips of coronary arteries were incubated for $2 \mathrm{~h}$ in $\left.{ }^{3} \mathrm{H}\right] 5-\mathrm{HT}\left(10^{-6} \mathrm{M}\right.$, adjusted specific activity, $2.8 \mathrm{Ci} / \mathrm{mmol}$ ) after which the strips were superfused as described for measurements of norepinephrine overflow. After an initial washout period of $120 \mathrm{~min}$, the superfusate was collected for 2-min intervals for measurement of the overflow of total radioactivity. Strips were stimulated three times for $6 \mathrm{~min}$ at $2 \mathrm{~Hz}(10 \mathrm{~V}, 0.2 \mathrm{~ms})$, $36 \mathrm{~min}$ apart. The second stimulation was done after 30 min pretreatment with, and in the presence of, tetrodotoxin $\left(10^{-7} \mathrm{M}\right)$. At the end of each experiment, the tritiated compounds were extracted from the tissue $(0.1 \mathrm{~N} \mathrm{HCl})$ and fractional release was calculated as for norepinephrine. The tritiated compounds overflowing the tissue immediately before and during electrical stimulation were collected in tubes containing $0.5 \mathrm{ml} 1 \mathrm{~N} \mathrm{HCl}$ with cold 5-HT $(10 \mu \mathrm{g} / \mathrm{ml})$, cold 5-hydroxyindole acetic acid (5-HIAA) $(10 \mu \mathrm{g} / \mathrm{ml})$ and ascorbic acid $(1 \mathrm{mg} / \mathrm{ml})$, pooled, and a portion was frozen for the later determination of intact $\left[{ }^{3} \mathrm{H}\right] 5$ HT (7). Evoked overflow of $\left[{ }^{3} \mathrm{H}\right] 5-\mathrm{HT}$ and $\left[{ }^{3} \mathrm{H}\right] 5$-HIAA was calculated as for $\left[{ }^{3} \mathrm{H}\right]$ norepinephrine.

Separation of 5-HT from 5-HIAA. Radiolabeled 5-HT was separated from its major metabolite, 5-HIAA by ion-exchange chromatography modified from Ahtee et al. (14) and Verbeuren et al. (7). In brief, the samples were thawed, the $\mathrm{pH}$ was adjusted to 6.5 with $0.5 \mathrm{~N}$ sodium carbonate, and the samples were passed through an Amberlite CG 50 column $(0.5 \times 8 \mathrm{~cm})$ in the sodium form at $\mathrm{pH}$ 6.5. The resin was then washed with $5 \mathrm{ml}$ water; the total effluent contained the 5-HIAA fraction. Radiolabeled 5-HT was eluted with $2 \times 5 \mathrm{ml} 6 \mathrm{~N}$ HCl-ethanol (1:1). By this procedure, the recovery of $\left[{ }^{14} \mathrm{C}\right] 5-\mathrm{HT}$ was $87 \pm 2.0 \%$ with a cross-contamination in the 5-HIAA fraction of $3.4 \pm 0.2 \%(n=5)$. The recovery of $\left[{ }^{14} \mathrm{C}\right] 5-\mathrm{HIAA}$ was $84 \pm 1.0 \%$ with a cross-contamination in the 5-HT fraction of $0.7 \pm 0.3 \%(n=5)$. The radiolabeled content or overflow was expressed as moles per milligram tissue, without correction for recovery.

Radioactivity measurements. 1-ml samples of the tissue extraction media, superfusate, and chromatographic fractions were added to $5 \mathrm{ml}$ Ready-Solv CP (Beckman Instruments, Inc., Fullerton, CA); radioac- 
tivity was counted in a liquid scintillation counter for 10 min or until counting error was $<5 \%$. Quenching was corrected for by the external standard method.

Drugs. The following pharmacological agents were used: acetylcholine chloride, cyproheptadine, 5-HT creatinine sulfate, 5-HIAA, 6-hydroxydopamine hydrochloride, indomethacin, 1-norepinephrine bitartrate, prostaglandin $F_{2 \alpha}$ (tris salt), tetrodotoxin (Sigma Chemical Co., St. Louis, MO); cocaine hydrochloride (generic); ketanserin bitartrate (Janssen Pharmaceuticals, Beerse, Belgium); 5-hydroxy-3 indolyl$\left[{ }^{14} \mathrm{C}\right]$ acetic acid (specific activity, $60 \mathrm{mCi} / \mathrm{mmol}$ ), 5-hydroxy (side chain-2 $-{ }^{14} \mathrm{C}$ ) tryptamine creatinine sulfate (specific activity, $55 \mathrm{mCi} /$ mmol, Amersham Corp., Arlington Heights, IL); 5- $\left[1,2-{ }^{3} \mathrm{H}(N)\right]-$ hydroxytryptamine creatinine sulfate (specific activity, $28.3 \mathrm{Ci} / \mathrm{mmol}$ ), L- $\left[7-{ }^{3} \mathrm{H}(N)\right]$ norepinephrine (specific activity, $24.8 \mathrm{Ci} / \mathrm{mmol}$, New England Nuclear, Boston, MA).

Drugs were dissolved in distilled water such that volumes $<0.5$ $\mathrm{ml}$ were added to organ chambers; concentrations are expressed as final molar concentration.

Statistical analysis. The data were expressed as means \pm SEM. Unless otherwise noted, statistical evaluation was by $t$ test for unpaired two-tailed comparisons between responses of different tissues; $\boldsymbol{n}$ refers to the number of dogs from which tissues were studied. The geometric means of the IC $_{50}$ 's are reported. $P$ values $<0.05$ were regarded as significant.

\section{Results}

Responses to electrical stimulation and exogenous norepinephrine of coronary arteries after exposure to platelets or 5-HT. After exposure of left circumflex coronary rings for $2 \mathrm{~h}$ to aggregating platelets, electrical stimulation from 0.5 to $4 \mathrm{~Hz}$ caused contractions that significantly exceeded the tension caused by prostaglandin $F_{2 \alpha}$ (Fig. 1). In contrast, relaxations in response to from 0.5 to $16 \mathrm{~Hz}$ occurred in rings exposed for the same duration to control solution or platelet supernatant. Electrical stimulation from 0.5 to $4 \mathrm{~Hz}$ also caused contractions of left circumflex rings exposed to 5-HT $\left(10^{-6} \mathrm{M}\right)$ for $2 \mathrm{~h}$. Higher frequencies of stimulation tended to cause relaxation of rings exposed to aggregating platelets or 5-HT, but these were significantly less than those observed in rings exposed to control solution or platelet supernatant. At 1 and $2 \mathrm{~Hz}$, the relaxations of rings exposed to platelet supernatant were significantly less than of those exposed to control solution.

After exposure to aggregating platelets or 5-HT, left circumflex rings relaxed in response to exogenously added norepinephrine. The sensitivity to norepinephrine was unchanged ( $\log \mathrm{IC}_{50}$ : before platelet exposure, $-6.9 \pm 0.1$; after exposure, $-6.9 \pm 0.1, n=9, P>0.5$ by paired $t$ test; before 5-HT exposure, $-7.3 \pm 0.1$; after exposure, $-7.2 \pm 0.1, n=5, P>0.5$ ).

Exposure of branch artery rings to 5-HT $\left(10^{-6} \mathrm{M}\right)$ for $2 \mathrm{~h}$ resulted in stimulation-induced contractions not significantly different from those observed in left circumflex rings; branch arteries exposed to control solution relaxed when stimulated (4 Hz: control solution, $-37 \pm 7.8(n=4) ; 5-\mathrm{HT}$ exposed, $+12 \pm 13(n=5)$, percent change in prostaglandin $F_{2 \alpha}$-induced tension).

Effect of serotonergic antagonists and cocaine on the responses of coronary arteries after exposure to platelets or 5-HT. The contractions in response to electrical stimulation observed in left circumflex rings exposed to platelets did not occur when the rings were treated with cyproheptadine $\left(10^{-6}\right.$ $\mathrm{M}, n=6)$ or ketanserin $\left(10^{-6} \mathrm{M}, n=8\right)$ after exposure to platelets. The relaxations in response to from 0.5 to $16 \mathrm{~Hz}$ in

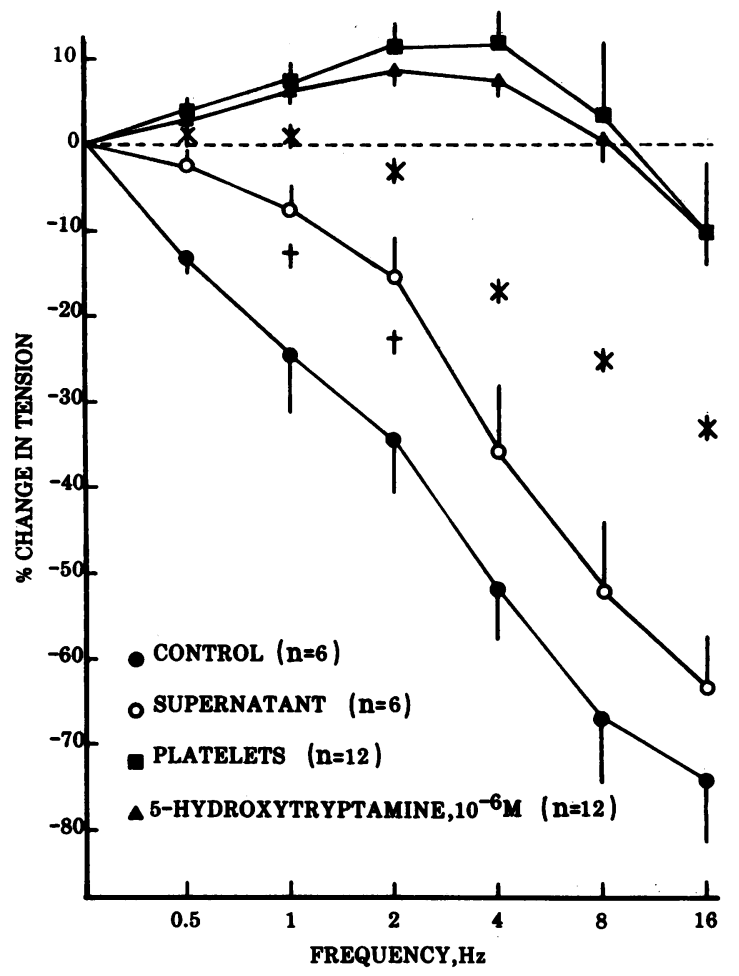

Figure 1. Effect of exposure for $2 \mathrm{~h}$ to aggregating platelets, platelet supernatant, 5-HT, or control solution on the subsequent response of canine left circumflex artery to electrical stimulation. Responses to from 0.5 to $16 \mathrm{~Hz}$ are expressed as percent change in tension induced by prostaglandin $F_{2 \alpha}$. Symbols denote means \pm SEM. ${ }^{*} \mathrm{Re}-$ sponses observed in rings exposed to aggregating platelets or 5-HT significantly different from those observed in rings exposed to the supernatant of the platelet suspension or control solution. +Significant difference in the relaxations of rings exposed to platelet supernatant as compared with those of rings exposed only to control solution.

the presence of either antagonist were not significantly different from those observed in rings exposed to control solution alone. In the presence of ketanserin, the stimulation-induced relaxations $(0.5-16 \mathrm{~Hz})$ of rings exposed to platelets were abolished by propranolol $\left(10^{-6} \mathrm{M}, n=2\right)$. Ketanserin $\left(10^{-6} \mathrm{M}\right)$ also abolished the stimulation-induced contractions of rings exposed to 5-HT $\left(10^{-6} \mathrm{M}\right)$ and resulted in frequency-dependent relaxations $(n=6)$.

Relaxation in response to exogenously added norepinephrine after exposure to platelets occurred with significantly increased sensitivity in the presence of ketanserin $\left(10^{-6} \mathrm{M}\right)$ but not of cyproheptadine $\left(\log \mathrm{IC}_{50}\right.$ : without antagonist, $-6.9 \pm 0.1$, $n=9$; ketanserin, $-7.4 \pm 0.1, n=6, P<0.025$; cyproheptadine, $-7.1 \pm 0.1, n=5, P>0.2)$. Ketanserin also significantly increased the sensitivity to norepinephrine after treatment with 5-HT $\left(\log \mathrm{IC}_{50}\right.$ : without antagonist, $-7.2 \pm 0.1, n=5$; ketanserin, $-7.7 \pm 0.1, n=5, P<0.025)$.

Treatment with cocaine $\left(3 \times 10^{-5} \mathrm{M}\right)$ significantly increased the relaxations of left circumflex rings in response to low frequency stimulation (Table I). The neuronal uptake inhibitor abolished the stimulation-induced contractions after exposure to 5-HT $\left(10^{-6} \mathrm{M}\right)$. When compared with the response of rings treated with cocaine alone, the response of rings exposed to 
Table I. Effect of Cocaine on Responses to Electrical Stimulation After Exposure to 5-HT*

\begin{tabular}{|c|c|c|c|c|c|c|}
\hline \multirow[b]{2}{*}{ Treatment } & \multicolumn{6}{|l|}{ Frequency $(\mathrm{Hz})$} \\
\hline & 0.5 & 1 & 2 & 4 & 8 & 16 \\
\hline $\begin{array}{l}\text { Control solution } \\
\text { throughout }(n=6)\end{array}$ & $0.79 \pm 0.25$ & $0.75 \pm 0.20$ & $0.77 \pm 0.18$ & $0.85 \pm 0.17$ & $0.96 \pm 0.21$ & $0.91 \pm 0.24$ \\
\hline $\begin{array}{l}\text { Cocaine alone } \\
\qquad(n=4) \ddagger\end{array}$ & $2.33 \pm 0.00 \S$ & $2.05 \pm 0.14 \S$ & $2.40 \pm 0.56 \S$ & $1.60 \pm 0.26 \S$ & $1.26 \pm 0.24$ & $1.08 \pm 0.14$ \\
\hline $\begin{array}{l}\text { 5-HT followed by } \\
\text { cocaine }(n=6)^{\prime \prime}\end{array}$ & $0.30 \pm 0.14 \pi$ & $0.25 \pm 0.18 \pi$ & $0.39 \pm 0.09 \pi$ & $0.41 \pm 0.09 \pi, * * *$ & $0.41 \pm 0.08 \uparrow, * * *$ & $0.39 \pm 0.08 \pi$ \\
\hline $\begin{array}{l}\text { Cocaine pretreatment } \\
\text { followed by } 5-\mathrm{HT} \\
(n=6) \ddagger \ddagger\end{array}$ & $1.04 \pm 0.27 \pi \cdot \S \S$ & $1.24 \pm 0.38 \S \S$ & $0.98 \pm 0.20 \pi \S \S$ & $0.90 \pm 0.17 \pi \cdot \S \S$ & $0.64 \pm 0.09 \pi$ & $0.57 \pm 0.10$ - \\
\hline
\end{tabular}

\begin{abstract}
* Responses of coronary rings to electrical stimulation were obtained before and after a 3-h treatment period. After the ring was contracted with prostaglandin $\mathrm{F}_{2 \alpha}\left(2 \times 10^{-6} \mathrm{M}\right)$, the relaxation in response to each frequency was measured as the change in tension. Values are means $\pm S E M$ of the ratio of the relaxation of the same ring to each frequency after the treatment period to that obtained initially in control solution. $\ddagger$ Treated with cocaine $\left(3 \times 10^{-5} \mathrm{M}\right)$ for at least $15 \mathrm{~min}$ before the second response. § Significantly greater than control solution. "Exposed to 5-HT $\left(10^{-6} \mathrm{M}\right)$ for $2 \mathrm{~h}$ followed by a 1 -h washout; cocaine $\left(3 \times 10^{-5} \mathrm{M}\right)$ was added during at least the last $15 \mathrm{~min}$ of the washout period. I Significantly less than cocaine alone. ** Significantly less than control solution. \# Cocaine $\left(3 \times 10^{-5} \mathrm{M}\right)$ was present 15 min before and during exposure to 5-HT $\left(10^{-6} \mathrm{M}\right)$ for $2 \mathrm{~h}$; cocaine was included during a 1-h washout. \$\$ Significantly greater than response of rings exposed to 5-HT followed by cocaine.
\end{abstract}

5-HT before treatment with cocaine was significantly inhibited at all frequencies studied. When rings were treated with cocaine before exposure to 5-HT, the inhibitory effect of 5-HT was significantly less.

Effect of exposure to 5-HT on stimulated overflow of radiolabeled norepinephrine. In coronary artery strips preincubated with $\left[{ }^{3} \mathrm{H}\right]$ norepinephrine, exposure to $5-\mathrm{HT}\left(10^{-6} \mathrm{M}\right.$, $2 \mathrm{~h})$ had no significant effect on the fractional release of tritiated compounds caused by $2-\mathrm{Hz}$ stimulation (control solution, $1.29 \pm 0.19 ; 5-\mathrm{HT}$ exposed, $1.34 \pm 0.27 \%$ release during $6 \mathrm{~min}, n=3$ ). Likewise, there was no significant difference in the stimulation evoked overflow of intact $\left[{ }^{3} \mathrm{H}\right]$ norepinephrine (control solution, 389 \pm 84 ; 5-HT exposed, $431 \pm 49 \mathrm{dpm} / \mathrm{mg}$ per $6 \mathrm{~min}$ ). Furthermore, the stimulation evoked overflow of $\left[{ }^{3} \mathrm{H}\right]$ norepinephrine after exposure to 5-HT or control solution represented a similar percentage of that caused by stimulation of the same strip before exposure (control solution, 78 \pm 9.8 ; 5-HT exposed, $72 \pm 9.5 \%$ ).

Influence of endothelium on responses to electrical stimulation after exposure to platelets. When rings denuded of endothelium were exposed to aggregating platelets for $2 \mathrm{~h}$, the responses to electrical stimulation were not significantly different from those shown in Fig. 1 for rings with intact endothelium ( $n=6$, data not shown).

Accumulation, metabolism, and overflow of radiolabeled 5-HT. During incubation with $\left[{ }^{14} \mathrm{C}\right] 5-\mathrm{HT}\left(10^{-6} \mathrm{M}\right)$ for $2 \mathrm{~h}$, similar quantities of $\left[{ }^{14} \mathrm{C}\right] 5-\mathrm{HT}$ and $\left[{ }^{14} \mathrm{C}\right] 5-\mathrm{HIAA}$ were accumulated by strips of coronary artery (Table II). Removal of the endothelium did not effect the accumulation of $\left[{ }^{14} \mathrm{C}\right] 5-\mathrm{HT}$ or its metabolism. Pretreatment with cocaine $\left(3 \times 10^{-5} \mathrm{M}\right)$ or 6-hydroxydopamine significantly inhibited the accumulation of $\left[{ }^{14} \mathrm{C}\right] 5-\mathrm{HT}$, but the tissue content of its metabolite was unaffected.

After incubation with $\left[{ }^{3} \mathrm{H}\right] 5-\mathrm{HT}\left(10^{-6} \mathrm{M}\right)$ and $2 \mathrm{~h}$ of superfusion, the basal overflow of tritiated compounds was composed of $4.5 \pm 1.4 \mathrm{fmols} / \mathrm{mg}$ per $6 \mathrm{~min}, 5-\mathrm{HT}$, and $35 \pm 5.3$ fmols/mg per $6 \mathrm{~min}, 5$-HIAA. Electrical stimulation $(2 \mathrm{~Hz})$ caused an increase in the fractional release of radiolabeled compounds (Fig. 2). The release of tritiated compounds was solely due to the stimulation evoked overflow of intact $\left[{ }^{3} \mathrm{H}\right] 5-$ $\mathrm{HT}$, as no significant evoked overflow of $\left[{ }^{3} \mathrm{H}\right] 5$-HIAA occurred (Fig. 3). Tetrodotoxin $\left(10^{-7} \mathrm{M}\right)$ abolished the fractional release of tritiated compounds and the overflow of $\left[{ }^{3} \mathrm{H}\right] 5-\mathrm{HT}$ evoked by electrical stimulation (Figs. 2 and 3).

\section{Discussion}

Responses to nerve stimulation. The method of transmural electrical field stimulation used in this study has previously

Table II. Effect of Different Treatments on the Accumulation of 5-HT by Coronary Arteries*

\begin{tabular}{lll}
\hline Treatment & {$\left[{ }^{14} \mathrm{C}\right] 5-\mathrm{HT}$} & {$\left[{ }^{14} \mathrm{C}\right] 5-\mathrm{HIAA}$} \\
\hline & pmol/mg tissue & pmol/mg tissue \\
Control & $2.7 \pm 0.3$ & $2.8 \pm 0.4$ \\
Endothelium denuded $\ddagger$ & $2.7 \pm 0.3$ & $3.0 \pm 0.2$ \\
Cocaine§ & $0.8 \pm 0.1 \pi$ & $2.5 \pm 0.4$ \\
Denervated" & $0.7 \pm 0.1 \pi$ & $2.6 \pm 0.4$ \\
\hline
\end{tabular}

* Radiolabeled compounds extracted from coronary segments incubated for $2 \mathrm{~h}$ with $\left[{ }^{14} \mathrm{C}\right] 5-\mathrm{HT}, 10^{-6} \mathrm{M}$. Values are mean $\pm \mathrm{SEM}$, $n=6$.

₹ Segments were denuded of endothelium before incubation.

$\S$ Segments were treated with cocaine $\left(3 \times 10^{-5} \mathrm{M}\right)$ for $15 \mathrm{~min}$ before and during incubation.

I Cocaine and denervation significantly decreased accumulation of $\left[{ }^{14} \mathrm{C}\right] 5-\mathrm{HT}$.

"Segments were denervated by treatment with 6-hydroxydopamine before incubation. 


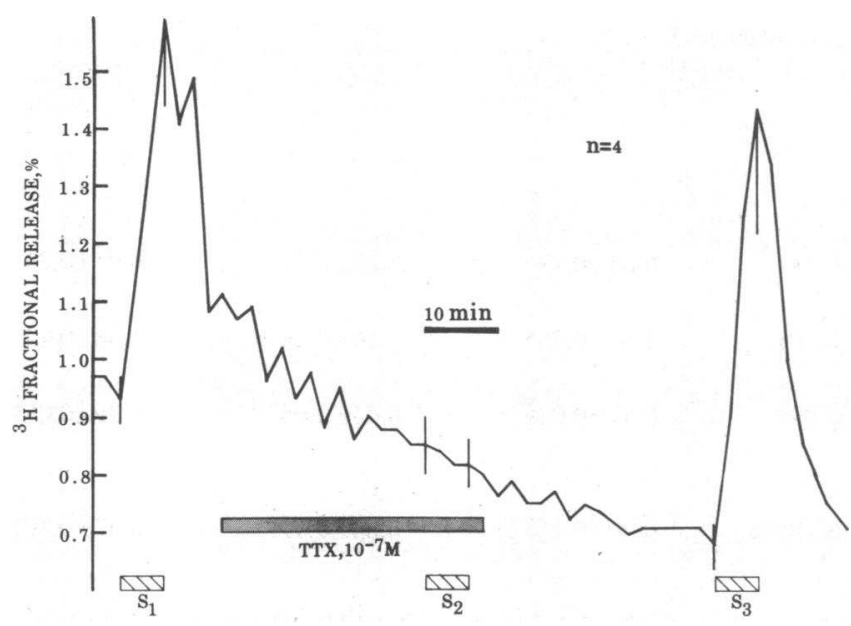

Figure 2. The fractional release of tritiated compounds from superfused coronary artery strips preincubated in tritiated 5-HT $\left(10^{-6} \mathrm{M}\right)$ and stimulated three times at $2 \mathrm{~Hz}$ for $6 \mathrm{~min}$. The tritiated compounds were collected for 2-min periods after $2 \mathrm{~h}$ of superfusing the strips with control solution. The fractional release is expressed as a percentage of the total tissue content of tritium calculated to be present before each 2-min collection. Standard errors are shown for the 2-min fraction just before each stimulation and for that during the last $2 \mathrm{~min}$ of stimulation. Before and during the second stimulation $\left(S_{2}\right)$, tetrodotoxin $\left(10^{-7} \mathrm{M}\right)$ added to the superfusate inhibited the stimulated overflow of tritium.

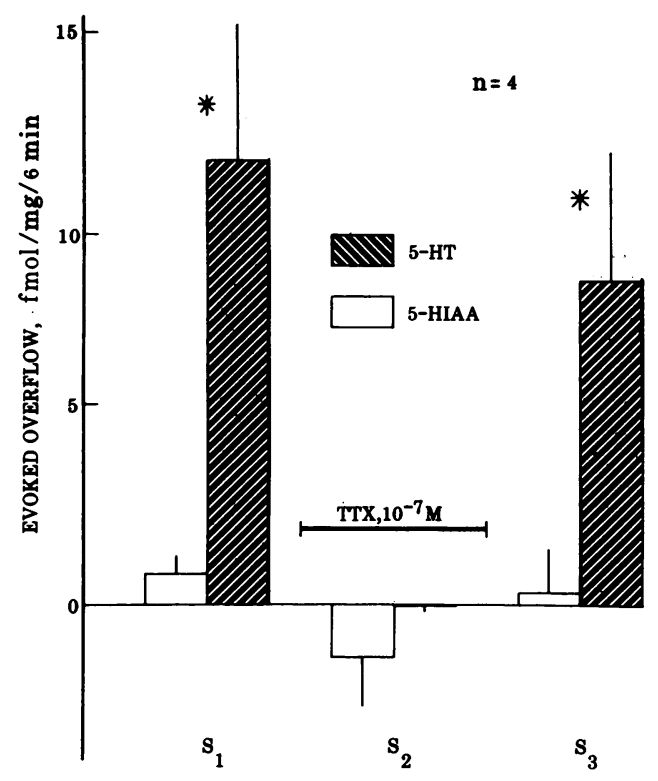

Figure 3. The evoked overflow of tritiated 5-HT and 5-HIAA that occurred during the three $2-\mathrm{Hz}$ stimulations shown in Fig. 2. The tritiated compounds were separated chromatographically, and the stimulation evoked overflow was determined as the contents of each compound (femtomoles per milligram tissue per $6 \mathrm{~min}$ ) present in the superfusate during the 6-min stimulation period minus that present during a 6-min period immediately before each stimulation.

Values are means \pm SEM. The asterisks refer to a significant overflow of tritiated 5-HT evoked by stimulation before and after washout of tetrodotoxin $\left(10^{-7} \mathrm{M}\right)$. Tetrodotoxin blocked the stimulated overflow of intact tritiated 5-HT. Stimulation evoked no significant overflow of 5-HIAA. been shown to cause relaxation of canine coronary arteries by virtue of a predominantly beta-adrenergic response to norepinephrine released from adrenergic nerve terminals (4). In this study, exposure of the coronary arteries to aggregating platelets and 5-HT was shown to reverse the response to electrical stimulation, resulting in stimulation-induced contractions. It is probable that 5-HT released from the aggregating platelets is responsible for the alteration in the response to electrical stimulation for the following reasons: $(a)$ The stimulationinduced contractions of rings exposed to 5-HT itself were similar to those of rings exposed to platelets. The concentration of 5-HT measured in the organ chamber after aggregation of platelets in a quantity similar to that in the present study was $\sim 0.4 \mu \mathrm{M}$ (2), and nearly equal to that used for comparison in this study. (b) Stimulation-induced contractions were not observed in vessels exposed to supernatant of platelet suspensions, suggesting that $5-\mathrm{HT}$ released during aggregation was responsible for the contractile response to electrical stimulation caused by platelets. (c) The serotonergic antagonists, cyproheptadine and ketanserin $(15,16)$, reversed the stimulation-induced contractions observed in vessels exposed to platelets to relaxations not different from those observed in control rings. The observation that rings exposed to platelet supernatant relaxed in response to electrical stimulation somewhat less than those exposed to control solution may indicate that some release of 5-HT into the platelet supernatant occurred before their aggregation, and that this lesser amount of the amine inhibits the response.

Cocaine potentiated the response to electrical stimulation of left circumflex artery rings, an effect that is probably due to its inhibition of norepinephrine re-uptake into sympathetic nerve endings $(17,18)$. The greater relaxation might explain why cocaine prevented the contractions caused by exposure to 5-HT. However, the response of rings stimulated in the presence of cocaine was inhibited by previous exposure to 5-HT, an effect that was lessened by treating the rings with cocaine before exposure to the amine. This suggests that the effect of 5-HT on the response to electrical stimulation in these studies depends on its neuronal uptake.

Responses to exogenous norepinephrine. The coronary arteries retained a normal response to exogenously added norepinephrine after exposure to aggregating platelets and 5-HT. This excludes the possibility that the altered response to nerve stimulation after exposure is due to a change in alpha- or betaadrenergic sensitivity of the coronary smooth muscle to norepinephrine released by the nerves. Ketanserin caused an increase in the sensitivity to norepinephrine to an extent similar to that shown previously to be caused by phentolamine (4), suggesting that this effect of ketanserin may be due to its known alpha-adrenoceptor blocking properties (15). Since cyproheptadine did not change the sensitivity to norepinephrine, it is probable that ketanserin antagonized the effects of exposure to platelets and 5-HT due to its serotonergic rather than its alpha-adrenergic properties. The lack of a significant role for alpha-adrenoceptors in the effects of exposure to 5-HT is further suggested by the contractions observed in branch arteries exposed to the amine, since this vessel is devoid of an alpha-adrenergic contractile mechanism (4).

Accumulation, metabolism, and release of 5-HT. The fact that cocaine inhibited the accumulation of radiolabeled 5-HT 
by coronary arteries suggests that the amine is concentrated in adrenergic nerve terminals. This is further suggested by the similar inhibition of accumulation observed in coronary arteries denervated by 6-hydroxydopamine (13). Despite the fact that most 5 -HT is apparently accumulated by adrenergic nerves, the metabolism of the amine, under the conditions studied, is largely extraneuronal, since the metabolism was unchanged in cocaine-treated or denervated arteries. Since an insignificant portion of the accumulation and metabolism of 5-HT occurred in endothelium, it is likely that the smooth muscle is largely responsible for its metabolism. The electrically stimulated release of radiolabeled 5-HT that was blocked by tetrodotoxin indicates that after its accumulation, the intact indoleamine is released by coronary adrenergic nerves.

5-HT as a false neurotransmitter. The present study suggests that, during exposure of coronary arteries to aggregating platelets, 5-HT is accumulated by adrenergic nerves and that the response of coronary arteries to subsequent electrical stimulation is abnormal due to neuronal release of the false neurotransmitter. The neurogenic contractions observed probably result from the neuronally released indoleamine acting at serotonergic receptors on the smooth muscle. When the contractions are blocked by ketanserin, the observed beta-adrenergic relaxations suggest that accumulated 5-HT is released as a cotransmitter with norepinephrine. In the absence of the serotonergic antagonist, the action of released norepinephrine is overcome by that of 5-HT.

It has been shown previously that 5-HT released by platelets acts at endothelial cell serotonergic receptors to inhibit the contractions of coronary smooth muscle that occur during exposure to aggregating platelets (1-3). Since neurogenic serotonergic contractions after exposure to platelets were not greater in coronary rings denuded of endothelium, neuronally released 5-HT apparently does not activate the endotheliumdependent inhibitory mechanism. This observation is similar to that made previously pertaining to the cholinergic neurotransmitter, acetylcholine; the exogenously added, but not the neuronally released transmitter, causes endothelium-dependent relaxation of coronary smooth muscle (10).

It is known that when most adrenergically innervated blood vessels are exposed during electrical stimulation to $5-\mathrm{HT}$, the release of norepinephrine and, consequently, the response of the tissue are inhibited $(19,20)$. The coronary arteries in this study were washed repeatedly after exposure to aggregating platelets and 5-HT; this was done to remove from the tissue free vasoactive substances that could have direct effects on neurotransmission. There was no difference in the stimulated overflow of $\left[{ }^{3} \mathrm{H}\right]$ norepinephrine from coronary strips after exposure to 5-HT, suggesting that 5-HT accumulated by and released from the nerves exerts little effect on neurotransmitter release. This further indicates that the released false neurotransmitter prevents relaxations in response to electrical stimulation predominantly by acting at smooth muscle 5-HT receptors.

Endothelial damage is known to result in platelet aggregation on subintimal connective tissue and smooth muscle, and release of platelet vasoactive substances at diseased sites on blood vessels is thought to play a role in angina pectoris and myocardial infarction $(21,22)$. In this study, neurogenic re- sponses of normal vessels were drastically altered after a brief exposure to numbers of platelets equivalent to that in plasma. In addition to the immediate contractile effects that 5-HT released by platelets could have on coronary smooth muscle (1-3), the present studies suggest that the accumulation of 5-HT by adrenergic nerves could result in their dysfunction. Conversion of adrenergic vasodilator nerves to a serotonergic vasoconstrictor function could contribute to coronary constriction during coronary thrombosis. If sympathetic nerve dysfunction persists, episodic neurogenic attacks of coronary vasospasm might occur at sites where platelets have aggregated.

\section{Acknowledgments}

The author thanks Katherine Boris for typing the manuscript and Kevin Zitnay for preparing the figures.

Dr. Cohen is supported by a Clinician-Scientist Award from the American Heart Association and his work was sponsored by National Institutes of Health grants HL 31607, HL 30668, and GRS S07 RR05487-12 and by grant 13-507-834 from the American Heart Association, Massachusetts Affiliate, Inc.

\section{References}

1. Cohen, R. A., J. T. Shepherd, and P. M. Vanhoutte. 1983. Inhibitory role of the endothelium in the response of isolated coronary arteries to platelets. Science (Wash. DC). 221:273-274.

2. Cohen, R. A., J. T. Shepherd, and P. M. Vanhoutte. 1983. 5-hydroxytryptamine can mediate endothelium-dependent relaxation of coronary arteries. Am. J. Physiol. 245:H1077-H1080.

3. Cohen, R. A., J. T. Shepherd, and P. M. Vanhoutte. 1984. Endothelium and asymmetrical responses of the coronary arterial wall. Am. J. Physiol. 247:H403-H408.

4. Cohen, R. A., J. T. Shepherd, and P. M. Vanhoutte. 1983. Prejunctional and postjunctional actions of norepinephrine at the sympathetic neuroeffector junction in canine coronary arteries. Circ. Res. 52:16-25.

5. Thoa, N. B., D. Eccleston, and J. Axelrod. 1969. The accumulation of $\mathrm{C}^{14}$-serotonin in the guinea pig vas deferens. J. Pharmacol. Exp. Ther. 169:68-73.

6. Junod, A. F. 1972. Uptake, metabolism, and efflux of $\mathrm{C}^{14}-5$ hydroxytryptamine in isolated perfused rat lungs. J. Pharmacol. Exp. Ther. 183:341-355.

7. Verbeuren, T. J., F. H. Jordaens, and A. G. Herman. 1983. Accumulation and release of $\left({ }^{3} \mathrm{H}\right)$-5-hydroxytryptamine in saphenous veins and cerebral arteries of the dog. J. Pharmacol. Exp. Ther. 226:579-588.

8. Paiva, M. Q., M. Caramona, and W. Osswald. 1984. Intra- and extraneuronal metabolism of 5-hydroxytryptamine in isolated saphenous vein of the dog. Naun.-Schmied. Arch. Pharmacol. 325:62-68.

9. Furchgott, R. F., and J. V. Zawadzki. 1980. The obligatory role of endothelial cells in the relaxation of arterial smooth muscle by acetylcholine. Nature (Lond.). 288:373-376.

10. Cohen, R. A., J. T. Shepherd, and P. M. Vanhoutte. 1984. Neurogenic cholinergic prejunctional inhibition of sympathetic betaadrenergic relaxation in the canine coronary artery. J. Pharmacol. Exp. Ther. 229:417-421.

11. Verbeuren, T. J., E. Coen, and P. M. Vanhoutte. 1977. Determination of ${ }^{3} \mathrm{H}$-norepinephrine and its metabolites in superfusate from isolated blood vessels. Arch. Int. Pharmacodyn. Ther. 227:171174. 
12. Verbeuren, T. J., W. J. Janssens, and P. M. Vanhoutte. 1978. Effects of moderate acidosis on adrenergic neurotransmission in canine saphenous veins. J. Pharmacol. Exp. Ther. 206:105-114.

13. Aprigliano, O., and K. Hermsmeyer. 1976. In vitro denervation of the portal vein and the caudal artery of the rat. J. Pharmacol. Exp. Ther. 198:568-577.

14. Ahtee, L., D. F. Sharman, and M. Vogt. 1970. Acid metabolites of monoamines in avian brain; effects of probenecid and reserpine. $\mathrm{Br}$. J. Pharmacol. 38:72-85.

15. Van Nueten, J. M., P. A. J. Janssen, J. VanBeek, R. Xhonneux, T. J. Verbeuren, and P. M. Vanhoutte. 1981. Vascular effects of

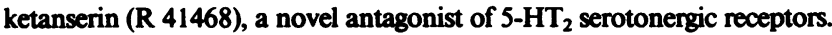
J. Pharmacol. Exp. Ther. 218:217-230.

16. Brazenor, R., and J. Angus. 1982. Actions of serotonin antagonists on dog coronary artery. Eur. J. Pharmacol. 81:569-576.

17. Vanhoutte, P. M., T. J. Verbeuren, and R. C. Webb. 1981. Local modulation of adrenergic neuroeffector interaction in the blood vessel wall. Physiol. Rev. 61:151-247.
18. Toda, N., and S. Hayashi. 1982. Responses of canine coronary arteries to transmural electrical stimulation and nicotine. Eur. $J$. Pharmacol. 80:73-81.

19. McGrath, M. A. 1977. 5-hydroxytryptamine and neurotransmitter release in canine blood vessels. Inhibition by low and augmentation by high concentrations. Circ. Res. 41:428-435.

20. Humphrey, P. P. A., W. Feniuk, and A. D. Watts. 1983. Prejunctional effects of 5-hydroxytryptamine on noradrenergic nerves in the cardiovascular system. Fed. Proc. 42:218-222.

21. Hirsch, P. D., L. D. Hillis, W. B. Campbell, B. G. Firth, and J. T. Willerson. 1981. Release of prostaglandin and thromboxane into the coronary circulation in patients with ischemic heart disease. $N$. Engl. J. Med. 304:685-691.

22. Maseri, A., A. L'Abbate, G. Baroldi, S. Chiercha, M. Marzilli, A. M. Ballestra, S. Severi, O. Parodi, A. Biagini, A. Distante, and A. Pesola. 1978. Coronary vasospasm as a possible cause of myocardial infarction. N. Engl. J. Med. 299:1271-1277. 